\title{
T Cells and Probably B Cells Arise from the Malignant Clone in Chronic Myelogenous Leukemia
}

\author{
A. A. Fauser, L. Kanz, K. J. Bross, and G. W. Löhr \\ Medizinische Klinik, Albert-Ludwigs-Universität, Freiburg, West Germany
}

\begin{abstract}
Bone marrow cells from a patient with $\mathbf{P h}^{\prime}$ positive chronic myelogenous leukemia in chronic phase were cultured for multilineage hematopoietic colonies (CFU-GEMMT), erythroid bursts, and granulocytic colonies. With CFU-GEMMT colonies, $T$ lymphocytes were identified by reaction with monoclonal antibodies Leu-5 and OKT-3; B cells were identified by reaction with $B_{1}$. All CFU-GEMMT colonies examined contained the $\mathbf{P h}^{\prime}$ chromosome. Recloned secondary colonies of $\mathrm{T}$ cells reacted with Leu-5 and OKT-3 and were $\mathrm{Ph}^{\prime}$ positive. This demonstrates that $\mathbf{P h}^{\prime}$ positive $\mathbf{T}$ lymphocytes were generated from the pluripotential stem cell of this patient. The presence of $B$ cells in the mixed colonies indicates that these may also be derived from the neoplastic clone.
\end{abstract}

\section{Introduction}

The presence of a single isoenzyme type of glucose-6-phosphate dehydrogenase (G-6-PD) ${ }^{1}$ in erythroblasts, granulocytes, megakaryocytes, and probably lymphocytes in women with chronic myelogenous leukemia heterozygous for this enzyme and the presence of the $\mathrm{Ph}^{\prime}$ chromosome in these cells provides evidence that all of these cell types may be derived from a neoplastic multipotential progenitor cell (1-3). Rearrangement of immunoglobulin genes in the cells of lymphoid blast crisis suggests that the neoplastic clone also produces cells of the Blineage containing cytoplasmic immunoglobulin $\mu$ chain (4). The origin of $\mathrm{T}$ lymphocytes and B lymphocytes during the chronic phase of this disease remains uncertain.

To determine the lineage of mature $T$ and $B$ cells in chronic myelogenous leukemia in chronic phase, we used the culture assay for primary mixed colonies (CFU-GEMMT) (59), and the technique of the clonal expansion of lymphoid elements (8) from such colonies to determine if lymphocytes

Address correspondence to Dr. Fauser, Division of Hematology, Royal Victoria Hospital, 687 Pine Avenue West, Montreal, Quebec, Canada H3A 1 Al.

Received for publication 29 August 1984 and in revised form 23 November 1984.

1. Abbreviations used in this paper: CFU-GEMMT, primary mixed hematopoietic colonies; EPO, erythropoietin; G-6-PD, glucose-6-phosphate dehydrogenase; PAP, peroxidase-anti-peroxidase; PHA, phytohemagglutinin; TCM, T cell conditioned medium.

J. Clin. Invest.

(C) The American Society for Clinical Investigation, Inc. $0021-9738 / 85 / 03 / 1080 / 03 \quad \$ 1.00$

Volume 75, March 1985, 1080-1082 grown from single colonies contained the $\mathrm{Ph}^{\prime}$ chromosome. We have recloned secondary colonies of lymphocytes derived from CFU-GEMMT colonies containing the $\mathrm{Ph}^{\prime}$ chromosome, we have identified the lymphocyte lineage of these cells using monoclonal lymphocyte markers $(10,11)$, and we have examined these for the $\mathrm{Ph}^{\prime}$ chromosome. The study demonstrates that such secondary colonies, characterized at $\mathrm{T}$ lymphocytes by such markers, contain the $\mathrm{Ph}^{\prime}$ chromosome and thus seem to be derived from the neoplastic clone.

\section{Methods}

Patient. Bone marrow was obtained from a 34-yr-old male with newly diagnosed chronic myelogenous leukemia in chronic phase before any treatment. The Philadelphia chromosome $\left(\mathrm{Ph}^{\prime}\right)$ was present in all examined metaphases.

Preparation of $T$ lymphocyte conditioned medium. Leu- 5 positive cells were separated with a FACS system from a patient with hemochromatosis (12), and conditioned medium was prepared. $10^{6} \mathrm{Leu}-5$ positive cells were incubated with $1 \%$ (vol/vol) human serum albumin (Sigma Chemical Co., St. Louis, MO), Iscove's modified Dulbecco's medium (Gibco Laboratories, Grand Island, NY), and 1\% phytohemagglutinin (PHA) (Wellcome Research, Triangle Park, NC) and the $T$ cell conditioned medium (PHA-TCM) was harvested after $4 \mathrm{~d}$ of incubation.

Preparation of cell suspensions. Bone marrow was aspirated into a heparinized syringe. Mononuclear cells of $d<1.077 \mathrm{~g} / \mathrm{ml}$ were obtained by centrifugation in Ficoll-Paque (Pharmacia Fine Chemicals, Piscataway, $\mathrm{NJ}$ ). In most studies, $2 \times 10^{-6}$ cells were added to thoroughly washed plastic dishes previously coated with Leu-5 and B1 antibody. After $1 \mathrm{~h}$ of incubation at $37^{\circ} \mathrm{C}$, the dishes were gently shaken and supernatants containing nonadherent cells were transferred to a washed B1 antibody coated plate. After $1 \mathrm{~h}$ of incubation, supernatants were doubly labeled with anti-Leu-1-PE (pan-T monoclonal, phycoerythrin conjugated) and anti-B1 + goat-anti-mouse Ig fluorescein isothiocyanate (B cell associated antigen, goat-anti-mouse IgG + IgM F(ab')2 antibody). These cells were analyzed using a FACS dual laser system. No stained cells were detected in these supernatants, indicating the absence of $T$ cells and B cells. The cells adhering to the dishes were tested for $T$ and $B$ cell characteristics by reaction with fluorescein isothiocyanatelabeled goat $F\left(a^{\prime}\right) 2$ anti-mouse IgG antibody (Cappel Laboratories, Cochranville, PA). $\sim 15 \%$ of the cells in the anti-Leu-5 coated dishes stained with anti-Leu-5, and 4-9\% of those adhering from the B1 coated dishes stained with anti-B1.

Culture conditions for mixed hematopoietic colonies. 200- $\mu \mathrm{l}$ aliquots of the nonadherent $T$ cell and $B$ cell depleted supernatant were admixed with $30 \%$ human fresh plasma, 5\% PHA-TCM (Leu-5 positive cells), Iscove's modified Dulbecco's medium, $2 \times 10^{-5} \mathrm{M}$ mercaptoethanol, and methylcellulose at a final concentration of $0.9 \%$ ( $\mathrm{vol} / \mathrm{vol})$ (12). 28 replicate dishes, each containing 6-7 $\times 10^{4}$ cells, were cultured. This density of cells was selected because multilineage colonies derived from bone marrow cells of G-6-PD heterozygous females revealed only one isoenzyme type when plated at this density (13). The incubation was performed at $37^{\circ} \mathrm{C}$ in a humidified atmosphere in $5 \% \mathrm{CO}_{2}$. After 
$4 \mathrm{~d}$ of incubation, $1 \mathrm{U}$ of erythropoietin (EPO) (Step III; Connaught Laboratories, Toronto, Canada) was added to 18 dishes of the cultures. Mixed hematopoietic colonies were identified at day 18 by their characteristic morphological appearance. These colonies contained cells with the red color typical of hemoglobin, admixed with colorless cells of various size (4-10). For further analysis, individual mixed hematopoietic colonies were removed by micropipette under sterile conditions from the cultures ( 8 ).

Recloning of hematopoietic colonies. Individually aspirated CFUGEMMT were divided into two parts. One portion was examined for the presence of $T$ cells or $B$ cells by using the peroxidase-antiperoxidase (PAP) slide technique. The second portion of each individual clone was replated as single cell suspensions in microtiter wells (Linbro Chemical Co., Hamden, CT). To stimulate lymphocyte proliferation, secondary plates contained 2.5\% PHA-TCM and 0.5\% PHA; no EPO was added. After $12 \mathrm{~d}$ of culture, the plates (Linbro Chemical Co.) were scored for secondary hematopoietic colonies. They were counted and individual colonies were aspirated by micropipette as described for primary colonies (8). The secondary colonies were again dissected into two portions. One portion of each individual colony was examined for cells expressing $T$ cell antigens using Leu- 5 and OKT3. The second portion was subjected to cytogenetic analysis (3).

$T$ cell and B cell markers on primary mixed hematopoietic colonies. Individual colonies were aspirated from the cultures by micropipette and were washed in Eppendorf micro test tubes. The pellets were resuspended after centrifugation $(5 \mathrm{~min}, 300 \mathrm{~g}$ ). The aliquots were either transferred onto poly-L-Lysine coated glass slides for staining of surface antigens, i.e., anti-Leu-5 and B1, by the PAP method using a glass slide technique described earlier $(14,15)$, or they were subcloned.

$T$ cell markers in secondary hematopoietic colonies. Individual secondary colonies were aspirated and washed as described. The pellet was resuspended and one portion was transferred onto the coated glass slides for surface antigen staining by the PAP method using anti-Leu5 or OKT3. The second portions were subjected to cytogenetic analysis $(3,16)$.

Cytogenetic analysis of hemopoietic colonies. Mixed hemopoietic colonies, erythroid bursts, granulocytic colonies, and aliquots derived from individual secondary colonies were subjected to chromosome analysis (3). 14 mixed colonies, 25 erythroid bursts, 25 granulocytic colonies, and 39 secondary $\mathrm{T}$ cell colonies were examined.

\section{Results}

T lymphocytes in multilineage colonies. CFU-GEMMT were obtained from cultures of bone marrow from this untreated patient with Philadelphia chromosome positive chronic myelogenous leukemia in chronic phase. Mixed colonies were observed in cultures supplemented with $1 \mathrm{U}$ of EPO and a smaller number of the absence of exogenously added EPO. The number of mixed colonies observed with EPO ranged from 3 to 7 per plate, and without EPO, ranged from 1 to 4 per plate. Individual mixed colonies were examined for the presence of $\mathrm{T}$ lymphocytes identified with the monoclonal antibody anti-Leu-5 or OKT3. Cells reacting with anti-Leu-5 ranged from 0 to $17 \%$ and were found in $\sim 35 \%$ of individual mixed colonies of the patient, irrespective of the presence or absence of EPO. The number of positive cells for the half split colony ranged from 0 to 71 cells. Individual erythroid bursts and granulocytic colonies cultured in the same dishes were found to be negative for $T$ cell markers.

$B$ cell associated antigen positive cells in multilineage colonies. The second portion of CFU-GEMMT was examined for cells staining for B cell associated antigens (B1). In 49 individual multilineage colonies, eight clones were identified that contained cells reacting with the $\mathrm{B} 1$ antibody. The number of cells positive for B1 ranged from 0 to 44 per examined colony.

Examination of the 21 mixed colonies that grew in the absence of EPO revealed three colonies with B1 positive cells, a proportion not different from those found in the presence of EPO.

Analysis of secondary $T$ cell colonies derived from multilineage colonies. A number of primary multilineage colonies formed secondary colonies upon replating in the presence of $2.5 \%$ PHA-TCM and $0.5 \%$ PHA. After $12 \mathrm{~d}$ of culture, individual secondary colonies were aspirated, washed, and split into two portions. The smaller portion of each individual secondary colony was analyzed for Leu-5 or OKT3 staining. All examined cells within each individual colony were either Leu- 5 or OKT3 positive.

Erythroid bursts and granulocytic colonies did not form secondary colonies upon replating and did not react with OKT3 or Leu-5 antibodies.

Cytogenetic analysis of secondary $T$ cell colonies derived from multilineage primary colonies. Cells derived from 39 individual secondary $\mathrm{T}$ cell colonies confirmed by the reaction with Leu-5 were subjected to cytogenetic analysis. The examination of 39 individual colonies revealed metaphases in 13 of them. In 10 preparations, one metaphase was observed, and in 3 preparations, two metaphases were identified. In addition, eight secondary colonies were examined by OKT3. All cells were OKT3 positive. All metaphases obtained from the secondary colonies were $\mathrm{Ph}^{\prime}$ positive, as were the metaphases obtained from individually examined mixed hematopoietic colonies, erythroid bursts, and granulocytic colonies.

\section{Discussion}

The development of an in vitro assay for CFU-GEMMT has provided a means of examining the progeny of single multipotent hemopoietic stem cells in human bone marrow when this is plated at a low density of cells. We have previously reported the presence of "pre-B" cells in such colonies from normal subjects (13), and of B cells from both normal subjects and patients with lymphocytic lymphoma (17). Different phenotypes of $\mathrm{T}$ lymphocytes have also been observed in such colonies, including $T$ cells reacting with the monoclonal antibodies OKT3 and OKT4 $(7,8)$, and cytotoxic T cells with OKT8 phenotype from patients with Hodgkins Disease (9).

We report the presence of subclones of cells with the $T$ lymphocyte phenotype from single colonies grown from a patient in the chronic phase of chronic myelocytic leukemia. All metaphases examined of these subclones contained the $\mathbf{P h}^{\prime}$ chromosome, as did metaphases of erythroid and myeloid cells. Cells with the B lymphocyte phenotype were also subcloned from mixed colonies from this patient but cytogenetic analysis of these is not available. This demonstrates that as in normal subjects, single stem cells from this patient could produce both lymphoid and myeloid progeny, and that the $\mathrm{Ph}^{\prime}$ chromosome was present in both hemopoietic and $\mathrm{T}$ lymphocyte progeny.

Evidence using G-6-PD subtypes and cytogenetic analysis has been published that in some patients with chronic myelocytic leukemia, lymphocytes may be derived from the same cell as the hemopoietic elements. The study provides direct proof of this, and suggests that the $\mathrm{Ph}^{\prime}$ chromosome probably is present in a very early transformed stem cell in this disease. 


\section{Acknowledgments}

This work was supported in part by Deutsche Forschungsgemeinschaft, and MRC (Canada) M8953.

\section{References}

1. Fialkow, P. J., R. J. Jacobson, and T. H. Papyannopoulou. 1977. Chronic leukemia: clonal origin in a stem cell common to the granulocyte, erythrocyte, platelet and monocyte/macrophage. Am. J. Med. 63:125-130.

2. Nowell, P. C., and D. A. Hungerford. 1960. A minute chromosome in human chronic granulocytic leukemia. Science (Wash. DC). 132:1497-1501.

3. Dubé, D., C. M. Gupta, D. K. Kalousek, C. J. Eaves, and A. C. Eaves. 1984. Cytogenetic studies of early myeloid progenitor compartments in $\mathrm{Ph}^{\prime}$ positive chronic myeloid leukemia (CML). Br. J. Haematol. 56:633-639.

4. Bakhshi, A., J. Minowada, A. Arnold, J. Cossman, J. P. Jensen, J. Whang-Peng, T. A. Waldmann, and S. J. Korsmeyer. 1983. Lymphoid blast crisis of chronic myelogenous leukemia represent stages in the development of B-precursors. N. Engl. J. Med. 309:826-831.

5. Fauser, A. A., and H. A. Messner. 1978. Granulo-erythropoietic colonies in human bone marrow, peripheral blood and cord blood. Blood. 52:1243-1248.

6. Fauser, A. A., and H. A. Messner. 1979. Identification of megakaryocytes, macrophages and eosinophiles in colonies of human bone marrow containing neutrophilic granulocytes and erythroblasts. Blood. 53:1023-1027.

7. Messner, H. A., C. A. Izaguirre, and N. Jamal. 1981. Identification of T-lymphocytes in human mixed hemopoietic colonies. Blood. 58: 402-405.

8. Fauser, A. A., and G. W. Löhr. 1982. Cloning of colonies positive for T-cell associated antigens derived from mixed hemopoietic colonies. Proc. Soc. Exp. Biol. Med. 170:220-224.
9. Fauser, A. A., H. A. Neumann, K. J. Bross, L. Kanz, and G. W. Löhr. 1982. Cytotoxic T-cell colonies derived from pluripotent stem cells (CFU-GEMM) of patients with Hodgkin's Lymphoma. Blood. 60:1317-1320.

10. Hara, H., S. Kai, M. Fushimi, S. Taniwaki, H. Ifuku, T. Okamoto, Y. Ohe, S. Fujita, K. Noguchi, A. Ranamaru, K. Nagai, and E. Inaela. 1981. Pluripotent erythrocytic and granulocytic hematopoietic precursors in chronic myelogenous leukemia. Int. J. Cell. Clon. 2:97-101.

11. Lepine, J., and H. A. Messner. 1983. Pluripotent hematopoietic progenitors (CFU-GEMM) in chronic myelogenous leukemia. Int. J. Cell. Clon. 2:97-101.

12. Fauser, A. A., B. A. Cooper, L. Kanz, and G. W. Löhr. Interaction of 3-deaza-adenosine, a phospholipid methyltransferase inhibitor, on the production of stimulatory activities of human peripheral T-cells. Exp. Hematol. (In press).

13. Powell, J. S., P. J. Fialkow, and J. W. Adamson. 1982. Polycythemia vera: studies of hematopoiesis in continuous long-term culture of human marrow. J. Cell. Physiol. 79(Suppl):79-85.

14. Fauser, A. A., L. Kanz, K. J. Bross, and G. W. Löhr. 1983. Identification of "Pre-B-cells" in multilineage hematopoietic colonies (CFU-GEMMT). Blood. 62(Suppl):43A.

15. Bross, K. J., G. M. Schmidt, K. G. Blume, W. E. Spince, and J. M. Farbstein. 1979. Conformation of bone marrow engraftment by demonstration of blood group antigens on red cell ghosts. Transplantation (Baltimore). 28:257-261.

16. Dubé, I. D., C. J. Eaves, D. K. Kalousek, and A. C. Eaves. 1981. A method for obtaining high quality chromosome preparation from single hemopoietic colonies on routine base. Cancer Genet. Cytogenet. 4:157-163.

17. Fauser, A. A., L. Kanz, and G. W. Löhr. Identification of Bcells in multilineage hematopoietic colonies (CFU-GEMMT) derived from patients with lymphocytic lymphoma. Proc. Natl. Acad. Sci. USA. (In press). 